\title{
A Topologically Robust Formation of Broadband Vortices Propagating at the Speed of Light
}

\author{
A. Zdagkas' ${ }^{1}$, V. Savinov ${ }^{1}$, N. Papasimakis ${ }^{1}$, Nikolay I. Zheludev ${ }^{1,2}$ \\ 1. Optoelectronics Research Centre \& Centre for Photonic Metamaterials, University of Southampton, SO17 1BJ, UK \\ 2. Centre for Disruptive Photonic Technologies \& TPI, SPMS, Nanyang Technological University, Singapore 637371
}

We show that Flying Doughnuts, the exact propagating solutions of Maxwell equations in the form of singlecycle toroidal pulses have complex and robust fine topological structure of spectrally broadband vortices and extended areas of energy backflow.

The study of the topology of electromagnetic waves provides insights into their fine-scale structure and can reveal information about their propagation dynamics. Typically, such studies are focused on vortices and polarization singularities of monochromatic light beams [1,2]. Here, we present a study of the singularities in the electromagnetic fields and Poynting vector of a single-cycle toroidal pulse, termed Flying Doughnut (FD). FD pulses are exact solutions of Maxwell's equations with known analytical form. Thus, such exotic forms of light are inherently stable under propagation and can be scrutinised at extraordinary level of detail. FDs propagate in freespace at the speed of light and their interactions with matter include non-trivial field transformations upon reflection from metallic and dielectric interfaces. The field configuration of the FDs is topologically similar to the toroidal dipolar excitations in matter, which allows to excite strong toroidal response and engage anapole modes (non-radiating configurations of electric and toroidal dipoles) in dielectric particles. It has been suggested that the longitudinal field components could be employed for the acceleration of charged particles.

In the FD pulse, energy is confined mainly in two toroidal-shaped lobes, each corresponding to a half-cycle, with azimuthal electric and poloidal magnetic fields. Examining in more detail the field configuration reveals a surprisingly complex fine-scale structure. The magnetic field of the pulse vanishes in 10 different regions (black dots in Fig. 1b). Along the propagation axis the magnetic field presents four saddle-like point singularities with the longitudinal component pointing towards (away from) and the radial component away from (towards) the singularity. Away from the propagation axis the magnetic field presents six vortex-like singularities. The singularities of the field are present also in the energy flow (see instantaneous Poynting vector in Fig. 1c). The Poynting vector at regions of high intensity is oriented along the propagation direction, while at regions of low intensity extended energy backflow is observed (white arrows in Fig. 1c). In addition, the Poynting vector vanishes on the $x y$ plane at $\mathrm{z}=0$ and along the propagation axis $(\rho=0)$, and on two spherical shells around the $\mathrm{z}$-axis (marked by the circular regions in Fig. 1c). The Poynting vector also presents 4 singular saddle-like points on the z-axis with forward (backwards) energy flow close to (away from) the origin, and 4 source/sink singularities away from the z-axis.

(a)

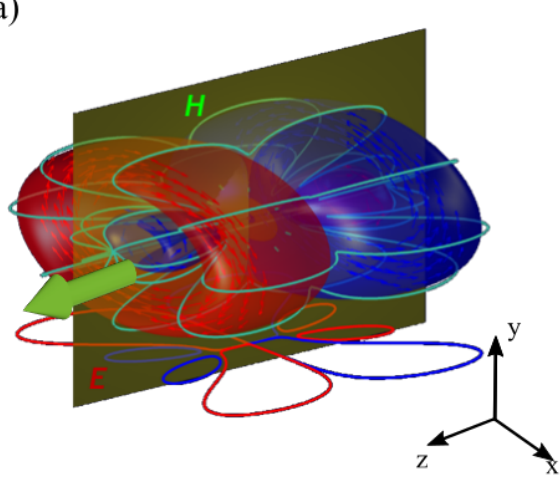

(b)

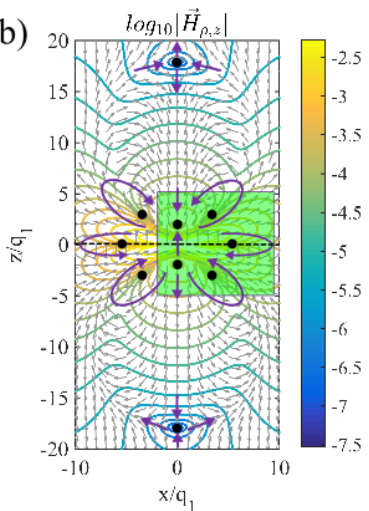

(c)

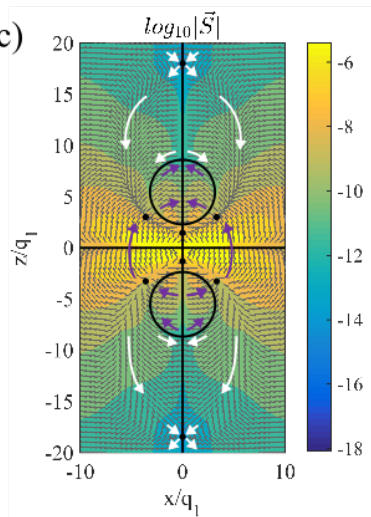

Fig. 1 Topology and singularities of a single-cycle transverse electric (TE) Flying Doughnut pulse at focus: (a) Isosurface of the electric consisting of two doughnut-shaped regions. Red and blue colors indicates the two half-cycles of the pulse. Green lines show the magnetic field winding around the electric field, while red arrows mark the direction of the latter. (b) Isoline and arrow plot of the logarithm of the magnetic field in the xz plane. Black dots indicate the zeros of the magnetic field. Magenta arrows mark the direction of the magnetic field and serve as guide to the eye. (c) Contour and arrow plots of the logarithm of the Poynting vector in the xz plane. Solid black lines, circles and dots mark the zeros of the Poynting vector. Magenta and white arrows indicate areas with forward and backward energy flow, respectively. Note that in regions of backward energy flow the Poynting vector takes values many orders of magnitude smaller than in the forward flow areas. In all panels, the propagation direction of the pulse is along the positive z-axis.

\section{References}

[1] J. F. Nye, M. V. Berry, "Dislocations in wave trains," Proc. R. Soc. A 336 165-90 (1974).

[2] M. R. Dennis, K. O’Holleran, M. J. Padgett, “Chapter 5 singular optics: optical vortices and polarization singularities,” Prog. Opt. 53, 293-363 (2009). 